International Journal of Instruction e-ISSN: 1308-1470 • www.e-iji.net

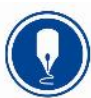

January $2020 \bullet$ Vol.13, No.1
p-ISSN: 1694-609X

pp. $855-868$

Received: 17/04/2019

Revision: 13/09/2019

Accepted: 25/09/2019

OnlineFirst:19/12/2019

\title{
Mathematics Teaching Using Word-Problems: Is it a Phobia!
}

\author{
Heba Bakr Khoshaim
}

Asst. Prof., Deanship of Educational Services, Prince Sultan University, Riyadh, Saudi

Arabia,hkhoshaim@psu.edu.sa

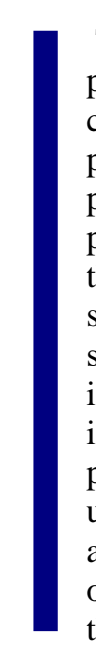

The mathematics education community has long emphasized applying wordproblem tasks in mathematics classrooms to foster understanding of mathematical concepts. As such, teachers of mathematics are eager to expose students to wordproblem mathematical tasks. However, students find such mathematical tasks particularly challenging, leading teachers to avoid implementing such wordproblems in classrooms. This study investigates the reasons behind the challenges that face students when working on word-problem tasks. The researcher applied a short questionnaire followed by interview sessions. The results revealed that students' inadequate skills and negative attitude towards mathematics led to increased difficulties in solving such tasks. Unaddressed, these difficulties result in increased stress and anxiety in the classroom, and eventually develop into a fear phobia of world problems. The findings also revealed that teachers must understand the importance of such tasks so they can commit the appropriate time and effort into its application. The study recommended having meaningful, handson, reader-friendly tasks that are relatable to students' lives and that are relevant to their interests.

Keywords: word-problem tasks, teacher's perspectives, challenges of solving wordproblems, attitude towards mathematics, mathematics achievements

\section{INTRODUCTION}

Through mathematics, scientists explore and explain natural phenomena. Yet, natural phenomena do not really come in mathematical representations. To address a phenomenon, scientists follow three phases: First, they translate the phenomenon from everyday language into what we call a mathematical task using the universal mathematical language. Then, they employ mathematical operations, algorithms, and theorems to solve the task. Then, they retranslate the solution to simple, comprehendible language that explains the phenomenon even to those with limited knowledge of mathematics. Mathematical tasks that address natural phenomena and require such phases are called word-problem mathematical tasks. 
In mathematics classrooms, the ability to solve word-problem tasks is an essential component of the learning outcomes of most mathematics courses. We should not only teach students how to implement routine procedures or apply algorithms, but also, we should show students the utility of using mathematics to address everyday challenges. In fact, working with word-problem tasks speaks to the "debate around the nature and relationship of mathematics, language and reality" (Gerofsky, 2010, p. 66) and supports that such tasks link the abstract subject of mathematics to real life. This is especially important if mathematics is taught to non-mathematics major students, where they need to sense the value of mathematics and the extent it is beneficial to their lives (National Council of Teachers of Mathematics [NCTM], 2000) to develop their appreciation for mathematics.

However, "the word-problem problem" (Ben Orlin, an Oakland-based high school teacher) is a well-known mathematics education challenge. For decades, mathematicians and mathematics educators acknowledged that students find such mathematical tasks challenging (Craig, 2001; Gerofsky, 1996; Schwanebeck, 2008; Seifi, Haghverdi, \& Azizmohamadi, 2012). In fact, Geary (1994) stated that students cannot solve word-problem tasks accurately although they can complete the computation part of the task. Moreover, Akhter, Akhtar, and Abaidullah (2015) and Doorman and maanen (2008) stated that solving algorithmic procedures is much easier for students than understanding the concepts behind them. Therefore, mathematics' teachers tend to avoid such tasks. Nevertheless, students majoring in mathematics find such tasks fruitful (Craig, 2001; Schoenfeld, 1985). Mathematics teaching and even assessments emphasize algorithms and procedures that aim for only numerical data.

Students prefer to only apply rules and procedures, or in other words, memorize the steps in order to solve a certain task. This, unfortunately, was shown to be insufficient to comprehend mathematical concepts (Boonen, de Koning, Jolles, \& van der Schoot, 2016). Hence, although mathematics teachers are encouraged to apply word-problem tasks to support the teaching and learning of mathematics, they face a dilemma between what is good for students and what is comfortable for them. To address the challenge, we should first understand the roots of it. However, the reasons behind the struggle with word-problem tasks have not been explored widely in the literature. Limited studies looked at the reasons behind the struggle with such tasks, but most of them considered school teachers, and only few of them (e.g. Craig, 2001) addressed the post-secondary level.

This study aims to explore mathematics teachers' perceptions about the reasons behind students' struggle with word-problem tasks. It also looks at teachers' pedagogical intention with regard to the use of word-problem tasks in their instruction especially if they are teaching non-mathematical major students. It is important to mention here that although few studies explored school teachers' perspectives with regard to not applying word-problems and why students do not find them valuable (e.g. Pearce, Bruun, Skinner, \& Lopez-Mohler, 2013; Seifi et al., 2012), this study will address universitylevel teachers' perspectives rather than school-level teachers. The study will investigate 1) what are the reasons behind students' struggle with word-problem mathematical tasks 
from teachers' perspectives, and 2) what (in their opinion) are the challenges of implementing word-problem tasks in higher education mathematics classrooms.

The mathematics education community has long acknowledged the word-problem challenge. Although many research results pushed for the implementation of such tasks in classrooms to comprehend mathematical concepts, very little has been done to understand why solving word-problem tasks is challenging for students, and why teachers tend to avoid applying such tasks. Previous studies emphasized the importance of applying word-problem tasks in mathematics classrooms to foster understanding (e.g. Boonen et al., 2016; Laster \& Charles, 2003) and to assess the level of comprehension (e.g. McCormick, 2010). Yet, very few studies looked at why students consider these tasks challenging and most of these studies considered only the secondary level. This study, on the other hand, looks at post-secondary level and investigates higher education mathematics teachers' perspectives.

\section{RELATED WORK}

\section{Attitudes Toward Mathematics}

As math educators, we, quite often, hear statements like: "I am not good at math"; I cannot do math", etc. It is almost unnegotiable that math is the most unfavourable subject in school (Dowker, Sarkar, \& Looi, 2016; Grežo \& Sarmány-Schuller, 2018). In fact, educators say that "no other school subject pushes emotional buttons the way math does" (Willis, 2010, p. 1). Attitudes toward mathematics affects achievements and vice versa. Meaning, students do not do well in mathematics classrooms because they do not like it (Dowker et al., 2016; Lipnevich, Maccann, Krumm, Burrus, \& Roberts, 2011); and because they do not do well in math classroom, they tend to be nervous and uncomfortable with it (Davadas \& Lay, 2018). Davadas and Lay (2018) and Willis (2010) claimed that such negative attitude might come from the parents rather than students. Because of its effect on performance, attitude toward mathematics cannot be ignored. In fact, productive disposition about mathematics is the 5th mathematical proficiency component (National Research Council, 2001). Willis argued that "the first step to success in math is a positive attitude. Yet that's the last thing we can expect from many of our students" (2010, p. 5). Attitude is important in all mathematics classrooms and is critically important if students are dealing with what they perceive challenging, such as the word-problem tasks.

\section{What is Word-Problem Tasks?}

Verschaffel, Greer, and De Corte (2000) defined word-problem mathematical tasks as "verbal descriptions of problem situation wherein one or more question are raised, the answer to which can be obtained by the application of mathematical operations to numerical data available in problem statement" (Seifi et al., 2012, p. 2923). In other words, mathematicians and scientists translate the matter to a mathematical task and then apply mathematical algorithms to solve it. In the last step, the concerned mater is solved by translating the mathematical solution to everyday language again. Basically, wordproblems addresses a matter in our lives and present the solution in simple everyday words. 


\section{Importance of Applying Word-Problem Tasks in Classrooms}

Solving word-problem tasks is one vital learning outcome in most mathematics courses and is considered an important skill that must be mastered by students (NCTM, 2000). Educators emphasize that teaching and learning mathematics should not be limited to only implementing procedures or applying algorithms. Working with word-problem tasks is a crucial phase to foster understanding of mathematical concepts (Boonen et al., 2016; Lambdin, 2003; Laster \& Charles, 2003). Such tasks can also be used to assess the level of comprehension to the mathematical concepts (McCormick, 2010). In fact, using word-problems in mathematics instruction was described as the "heart of mathematics" (Cankoy \& Özder, 2011, p. 92).

By solving word-problem tasks, students can sense the beauty of using mathematics to

address everyday challenges and hence, appreciate it and value it further. It was shown that applying word-problem tasks in classrooms shows students how mathematics is connected to our daily life (Akhter et al., 2015), which increases their level of motivation (Lambdin, 2003), improves disposition (NCTM, 2000) and empowers the role of mathematics (Attard, 2011). One of the teachers in Gerofsky's study argued about why he focusses on word-problem tasks in calculus classes. "I would like them to be able to see mathematics in or project mathematics onto situations around them" (Gerofsky, 2004, p. 55). Besides, lists of theorems or proofs are not useful or important if we cannot sense how they can make our life easier. In fact, theorems, proofs, and algorithms are considered boring, which will make students not only perceive mathematics classes as difficult, but also as tedious and useless. Doing so will only support the myth that math is a rigid, and pathetic subject.

\section{Important, Yet Challenging: It is a Phobia!}

The challenge of solving word-problem tasks in mathematics classrooms is not a new topic in mathematics education. In fact, discussing such matter "has been a reoccurring topic in professional literature for the last century" (Seifi et al., 2012, p. 2923). Students from all ages, grades, and cultures face the word-problem tasks challenge. Such challenge is independent of the language or culture; previous literature suggested that whether the task is in Arabic (Al-Onazi, 2011), English (Craig, 2001) or Turkish (Cankoy \& Özder, 2011), for example, the challenge with such tasks exists.

On the other hand, continuous struggle in word-problems results in frustration, and low self-esteem (Schwanebeck, 2008). Additionally, low self-esteem affects students' willingness to solve such tasks as they view them as confusing, puzzling problems where only smart students can solve them (Lambdin, 2003). As argued above, the ability to solve word-problem tasks fosters understanding of mathematical concepts, which will improve students' confidence level, motivation, and engagement. Hence, students will be trapped in a cycle of low self-esteem, lack of confidence, and unwillingness and inability to solve world-problem tasks.

Previous research looked at the factors that could hinder students' ability to solve wordproblem tasks. Few factors have been identified. While Cankoy and Özder (2011) 
emphasized on the format of the task, Craig (2001) and Pearce et al. (2013) argued that the linguistic abilities of students are a major factor. On the other hand, students' previous knowledge and skills was discussed. Craig (2001) perceived students' previous mathematical skills as essential components. Additionally, the literature suggested few solutions. For example, Davis-Dorsey, Ross, and Morrison (1991) claimed that the familiarity with the context might ease the challenge. In addition, Eric (2011) argued that introducing the task within the right environment will improve students' attitude toward the word-problem, which in return will improve their ability to solve the task.

\section{METHOD}

The goal of this study is to examine mathematics teachers' perceptions and pedagogical intentions regarding the use of word-problem tasks when teaching mathematics. As such, the research focus is university faculty members teaching mathematics courses to non-mathematics major students. This research uses the Case Study approach to address one private university in the Kingdom of Saudi Arabia. The sample of the study was mathematics teachers at this university. Participants' responses answer the following research questions: 1) what are the reasons behind students' struggle with word-problem mathematical tasks from teachers' perspectives, and 2) what, in their opinions, are the challenges of implementing word-problem tasks in higher education mathematics classrooms?

Data collection was completed in two phases; in Phase I, the researcher used previous literature results to identify factors that could be behind the challenge facing students when dealing with word-problem tasks and that could hinder the process of implementation in classrooms. The factors can be classified in three categories: 1) wordproblem characteristics; 2) students' characteristics; and 3) the educational/classroom settings. The researcher then developed a short questionnaire (Appendix I) based on these factors. Next, the researcher piloted the questionnaire with two colleagues using the think-aloud approach to ensure clarity, simplicity, and appropriateness of the items. The questionnaire was then employed to all mathematics faculty members in a private university. A total of 18 mathematics teaching staff (eight females and 10 male) received the questionnaire. Ten of the 18 faculty members have a PhD degree in mathematics.

In Phase II, the researcher used the questionnaire results to develop a semi-structured interview protocol and applied it on selected faculty members. A total of four faculty members were interviewed. All interviewees have been teaching mathematics courses at the college level for over 10 years. Two of the interviewees hold a $\mathrm{PhD}$ degree in mathematics whereas the other two hold a MSc degree. Moreover, two of the participants have participated in presenting professional workshops given to math teachers. Also, two of them held the Higher Education Academy certificate. It is also important to mention here that three of them taught in both private and public universities. The interviews were completed between Dec 6, 2018 and March 15, 2019.The interviews took place in the university campus. All interviews lasted for about 30 minutes. It is important to mention here that the Institutional Review Board approval was obtained prior to collecting data. The researcher realized that the sample size of the questionnaire is small. However, the results of the questionnaire were only used to guide 
the interview process and to develop the interview protocol. Only descriptive statistics is used from Phase I.

\section{FINDINGS}

\section{Results of Phase I:}

The questionnaire was administered to 18 mathematics teachers. Responses were received from 15 teachers. The descriptive statistics of the sample show that $60 \%$ of the sample hold a $\mathrm{PhD}$ degree whereas $40 \%$ hold a master's degree in mathematics. The teachers taught various mathematics courses including calculus, linear algebra, and finite mathematics and more that $70 \%$ of them have over ten years of teaching experience.

The survey results revealed that $80 \%$ of the teachers believe that the linguistic, vocabulary and structure of the word-problem are factors that could make the wordproblem challenging. Around $70 \%$ of them argued that non-academic factors such as students' dispositions toward mathematics, confidence level and persistence to solve the problem influence students' ability to solve such tasks. Moreover, more than $80 \%$ of the teachers think that student's English language proficiency is one of the obstacles to solve word-problem tasks. On the other hand, teachers agreed (around 90\% of them) that the allocated time of the class and the academic level of students will affect their decision to apply word-problems when teaching.

\section{Results of Phase II}

Four mathematics teachers were interviewed, two master holders and two PhD holders. All interviewees had more than 10 years of teaching experience. First, the researcher transcribed all interviews. The interviewees were given codes: Participant A, B, C, and D. Then, the researcher read through the transcripts and highlighted the quotes that reflect teachers' opinions.

\section{Terror caused by word-problem tasks}

One of the interviewees actually called it "a phobia!". For Participant A "it is a phobia [for students]!". "They are afraid of the word-problem"—as explained by Participant D. So, failure to solve such problems is affecting students emotionally and mentally, and eventually their ability to solve the task. Students' frustration is transferred to teachers. Participant D stated that the stress from explaining such tasks with very little rewarding from students is disappointing. "Sometimes I just want to scream" (Participant D).

The interviewees agreed on some factors that are behind the challenge of solving wordproblem tasks:

\section{Reasons behind students' struggle}

Although the participants argued about various reasons, they all focused on students' previous experience. "Students are not used to think...they only memorize, so they cannot analyse...even good students cannot do it" (Participant A). Participant B summarized the reasons behind the challenge by saying "new concepts...they are not given this in school". On the other hand, Participant A explained: "there is a gap 
between high school and university level...they are weak". Furthermore, Students' lack of readiness could be related to the teacher. Participant A and Participant $\mathrm{C}$ were both involved in professional development workshops that addressed school teachers. Participant A stated that "...... in the workshop we did, when it is a word-problem, the teacher cannot do it" [meaning, cannot solve the word-problem]. "When I did the workshop, the curriculum has word-problems, however, it is ignored! The problem is not in the curriculum but in implementing the curriculum". However, "students will be trained on word-problems when it is time to prepare for the Qudorat test ${ }^{1}$ " (Participant C).

In addition, Participant D pointed out that apart from calculations, there are additional skills needed to solve word-problem tasks. She stressed on the three steps to solve any word-problem and that if a student failed in any step, then he/she cannot solve it. Participant D also emphasized that students' basic skills of math are weak.

\section{Reasons NOT behind students' struggle}

Phase I participants suggested that the language constrain might be one of the essential reasons behind students' struggle. However, all four interviewees disagreed! "The language is not an issue... because if they are dealing with tasks in their mother-tongue, still they won't do it" (Participant A). Participant B approved what Participant A stated and emphasized that students need to translate so their language should be good, but this is not the main reason. Participant $\mathrm{C}$ added: "some are weak in English but can do it, but other native speakers cannot,..this is a problem even if it's (the task) in Arabic" [their native language]. Participant D also argued against the language challenge. "We have international students ...you know, they still have an issue...language is an issue but it is not the main reason".

The interview results revealed that the word-problem challenge is not related to the culture or race. Participant A reflected that she taught two years in Lebanon. She added: "they also have a problem" to solve word-problem tasks. On the other hand, while the class timings were pointed out to be a constrain, Participant D emphasized that "class timing is not an issue, it will be harder on me (the teacher) only".

\section{Reasons behind teachers' resistance}

The time factor appeared to be a major reason behind teacher's reluctant to apply wordproblem tasks. "At least 20 minutes will be lost.....the time is a big issue" (Participant D). Participant A reflected that the "materials are already a lot; how do we add wordproblem on top".

On that other hand, the teacher might resist to apply word-problem tasks because they might think that it is probably not worth it. "For some tasks, word-problem will complicate the concept we are addressing...sometimes the topic is hard, so also we

\footnotetext{
${ }^{1}$ Qodorat is the General Aptitude Test that all high school graduates must take as an admission condition to all higher education in the kingdom
} 
apply word-problem on it, it is a disaster" (Participant D). On the other hand, they might believe that students are not ready, and they cannot accomplish what is needed. "I taught at SSU (a public university), students were more academically ready than here, so I applied word-problem more" (Participant C).

\section{Suggested solutions}

Participant B said that students need to imagine the case; students must "understand the question and translate it to math, the first stage is very difficult, if he can do it, it is done". Hence, Participant B emphasized that it is important to "have question from their specialty... you know related to their major or their life so they can understand". Another solution is to "have live activities, live projects, you know... have dollars and work as a bank accountant and work with load". On the other hand, Participant D highlighted that students need to read the task several times and divide the problem into sections. It is important also "to translate each section to math". In that, Participant C stated that students are always encouraged to draw a picture or reward the tasks so they can visualize the context. "They should understand the problem so they can translate...Critical thinking is important" (Participant C). Participant D emphasized that students should "be able to write the plan to solve the problem then applying the rules...they need to connect the problem to math....and they need to make logic of the solution".

\section{DISCUSSION AND RECOMMENDATION}

The findings of this research shed light on the most important factors behind students' struggle in solving word-problem mathematical tasks from teachers' perspectives. Moreover, the results of this research informed the mathematics education society on the approaches that could be used to address the challenges behind implementing wordproblem tasks in mathematics classrooms.

It is interesting to realize that teachers in both phases do not see class size or setting as hinders to applying word-problem tasks. It is also noticeable that not all factors identified in Phase I were highlighted in Phase II. For example, while teachers in Phase I believed that language proficiency is among the challenges that hinder the ability to solve word-problems, the interviewees disagreed and argued that the language challenge or the culture differences is a minimum factor. This coincides with previous research that minimized the effect of the language/culture factor (Al-Onazi, 2011; Cankoy \& Özder, 2011; Craig, 2001). Nevertheless, Gerofsky (2010) concurred with Phase I results and emphasized the unambiguity of a language is never guaranteed and such ambiguity is the main reason behind the word-problem challenge. Additionally, while the interviewees did not see the structure of the task as a critical factor, Phase I participants and previous research findings - such as Cummins, Kintsch, Reusser, Weimer (1988) and Seifi et al. (2012) —identified the structure of the word-problem as one of the reasons behind the challenge of word-problems.

The survey and interviews findings agreed on some reasons behind the problem. These reasons coincide with previous literature in most cases. For example, Participant D suggested that the weakness of students' previous skills is a major obstacle. Craig 
(2001) emphasized that it is not only the lack of skills but sometimes students come to college with wrong skills/habits which will hinder the learning process, especially students' problem-solving skills. In addition, negative attitude and the lack of selfconfidence was found to effect performance in mathematics classrooms (Willis, 2010). This was highly suggested by the findings of this study with $70 \%$ of the survey respondents and most interviewees think of attitude as a huge factor emphasizing that the fear to fail is affecting the ability to solve such tasks.

Moreover, the suggestion from Participant B about addressing the environment or the content of the task was suggested in previous research (Cankoy \& Özder, 2011; DavisDorsey et al., 1991; De Corte, Vershaffel, \& De Win, 1989; Eric, 2011; Seifi et al., 2012). The literature also argued about the importance of linking the tasks to what students love and making it related to their fields (Willis, 2010). Participant B cannot agree more. In fact, Participant B suggested to have hands-on, live activities that are related to what students love. Likewise, Seifi et al. (2012) concluded that students can draw a picture to be able to visualize the task, which what was suggested by Participant C. Likewise, Al-Onazi (2011) argued about the importance of rewarding the task to simplify it, which what was highlighted by Participant $\mathrm{C}$.

It is important to note that some teachers think that the time spent on such tasks might be lost (or that the time spent is not worth it). Some participants pointed out to the amount of syllabus they need to cover and that implementing such tasks might influence their goal. Such comments suggest that the negative attitude does not only influence students, but also teachers, which will influence their decision to implement such tasks.

\section{CONCLUSION AND IMPLICATIONS}

The number of previous findings that support the importance of applying word-problem tasks in mathematics classrooms is tremendous. The amount of literature that emphasized the challenge of applying such tasks is also very large. However, limited research looked at why this challenge occurs or whether some academic or nonacademic factors enlarge the difficulties. Moreover, most previous investigations addressed secondary education and did not consider higher education classrooms. The results of this study shed light on some of the factors that contribute to the challenges that are facing students when working with word-problem tasks and suggested some possible solutions that might address these challenges.

The findings of this study suggest that educators - before implementing word-problem tasks - must ensure that students are equipped with the required skills. In addition, the findings recommend to have meaningful, hands-on, reader-friendly tasks that are relatable to students' lives and that are relevant to their interests. This in return will help in fostering a positive attitude towards the tasks. Furthermore, teachers must believe in the importance of such tasks and commit the required time and efforts into its application. Hence, managements of higher education institutions along with mathematics educators must involve and inform teachers with the pedagogical approaches that foster the process of learning - such as the implementation of wordproblem tasks - and ensure that teachers are aware of and committed to the importance 
of such approaches. Professional development programs must not only focus on teachers' mathematical knowledge, but also address their beliefs and commitments to the significance of certain pedagogical tools.

\section{REFERENCES}

Akhter, N., Akhtar, M., \& Abaidullah, M. (2015). The perceptions of high school mathematics problem solving teaching methods in mathematics education. Bulletin of Education and Research, 37(1), 1-23

Al-Onazi, M. (2011). Effectiveness of a recommended workshop to improve mathematics teachers' ability to problem solving strategies to improve students' abilities to mathematical thinking, problem solving and disposition toward mathematics at Araar (Unpublished doctoral dissertation). Umm Alqurra University.

Attard, C. (2011). My favourite subject is maths. For some reason, no-one really agrees with me: Student perspectives of mathematics teaching and learning in the upper primary classroom. Mathematics Education Research Journal, 23, 363-377.

Boonen, A. J. H., de Koning, B. B., Jolles, J., \& van der Schoot, M. (2016). Word problem solving in contemporary math education: A plea for reading comprehension skills training. Frontiers in Psychology, 7, 1-10. doi.org/10.3389/fpsyg.2016.00191.

Cankoy, O., \& Özder. H. (2011). The influence of visual representations and context on mathematical word problem solving. Pamukkale Uni. J. of Education, 30(2), 91-100.

Craig, T. (2001). Factors affecting students' perceptions of difficulty in calculus word problems (Unpublished master thesis). Cape Town University.

Cummins, D., Kintsch, D., Reusser, K., \& Weimer, R. (1988). The role of understanding in solving word problems. Cognitive Psychology, 20, 439-462.

Davadas, S. D., \& Lay, Y. F. (2018). Factors affecting students' attitude toward mathematics: A structural equation modeling approach. Eurasia J. of Mathematics, Science, and Technology Education, 14(1), 517-528. doi: 10.12973/ejmste/80356.

Davis-Dorsey, J., Ross, S. M., \& Morrison, G. R. (1991). The role of rewording and context personalization in the solving of mathematical word problems. Journal of Educational Psychology, 83(1), 61-68. http://dx.doi.org/10.1037/0022-0663.83.1.61.

De Corte, E., Vershaffel, L., \& De Win, L. (1989). Teaching word problem in the primary school: What research has to say to the teacher? In B. Greer \& G. Mulhern (Eds.), New development in teaching mathematics (pp 85-106). London: Routledge.

Doorman, M., \& Van maanen, J. (2008). A historical perspective on teaching and learning calculus. Australian Senior Mathematics Journal, 22(2), 4-14.

Dowker, A., Sarkar, A., \& Looi, C. Y. (2016). Mathematics anxiety: What have we learned in 60 years? Frontiers in Psychology, 7, 1-16. doi.org/10.3389/fpsyg.2016.00508. 
Eric, C. C. M. (2011). Primary 6 students' attitudes towards mathematical problemsolving in a problem-based learning setting. The Mathematics Educator, 13(1), 15-31.

Geary, D. C. (1994). Children's mathematical development: Research and practical applications. Washington, DC: American Psychological Association.

Gerofsky, S. (1996). A linguistic and narrative view of word problems in mathematics education. For the Learning of Mathematics, 16(2), 36-45.

Gerofsky, S. (2004). A man left Albuquerque heading East: Word problems as genre in mathematics education. New York: Peter Lang.

Gerofsky, S. (2010). The impossibility of 'real-life' word problems (according to Bakhtin, Lacan, Zizek and Baudrillard) discourse. Studies in the Cultural Politics of Education, 31(1), 61-73. doi: 10.1080/01596300903465427.

Grežo, M., \& Sarmány-Schuller, I. (2018). Do emptions matter? The relationship between math anxiety, and problem solving ability. Studia Psychologica, 60(4), 226244. doi: 10.21909/sp.2018.04.764.

Lambdin, D. V. (2003). Benefits of teaching through problem solving. In F. K. Laster, Jr, \& R. I. Charles (Eds.), Teaching mathematics through problem solving: Prekindergarten-grade 6 (pp. 3-10). Reston, VA: National Council of Teachers of Mathematics.

Lipnevich, A. A., Maccann, C., Krumm, S., Burrus, J., \& Roberts, R. D. (2011). Mathematics attitudes and mathematics outcomes of U.S. and Belarusian middle school students. Journal of Educational Psychology, 103(1), 105-118. doi:10.1037/a0021949.

McCormick, K. (2010). Experiencing the power of learning mathematics though writing. Undergraduate Mathematics Preparation of School Teachers: The Journal, 4(2), 1-8.

National Council of Teachers of Mathematics (2000). Curriculum and evaluation standards for school mathematics, Reston, VA: National Council of Teachers of Mathematics

National Research Council. (2001). Adding it up: Helping children learn mathematics. Washington, DC: National Academy Press.

Laster, F. K. (Jr), \& Charles, R. I. (2003). Teaching mathematics through problem solving: Prekindergarten-grade 6. Reston, VA: National Council of Teachers of Mathematics.

Pearce, D. L., Bruun, F., Skinner, K., \& Lopez-Mohler, C. (2013). What teachers say about student difficulties solving mathematical word problems in grades 2-5. International Electronic Journal of Mathematics Education 8(1), 3-19.

Seifi, M., Haghverdi, M., \& Azizmohamadi, F. (2012). Recognition of students' difficulties in solving mathematical word problems from the viewpoint of teachers. 
Journal of Basic and Applied Scientific Research, 2(3), 2923-2928.

Schoenfeld, A. (1985). Mathematical problem solving. Orlando, Florida: Academic.

Schwanebeck, T. (2008). A study of the summarization of word problems (Unpublished mater theses). Retrieved from Summative Projects for MA Degreehttp://digitalcommons.unl.edu/mathmidsummative/39.

Verschaffel, L., Greer, B., \& De Corte, E. (2000). Making sense of word problems. Lisse, The Netherlands: Swets \& Zeithinger.

Willis, J. (2010). Learning to love math: teaching strategies that change student attitudes and get results. Alexandria, VA: ASCD.

\section{APPENDIX I}

\section{QUESTIONNAIRE ITEMS}

\section{Section I: General Information}

\section{Gender}

$\begin{array}{ll}\circ & \text { Male } \\ \circ & \text { Female }\end{array}$

Highest academic qualifications

- Master

- Doctoral

\section{Years at PSU}

$0-5$

5-10

more than 10 years

Courses you have taught at PSU (please click all that apply)

Calculus I

Calculus II

Geometry

Numeral Analysis

Business Calculus

Liner Algebra

Finite Mathematics

Differential Equations

Engineering Mathematics

\section{Section II:}

It was reported in the literature that students find working on word-problems mathematical tasks challenging.

- The following are some elements related to the problem that have been reported in the literature as factors behind the difficulties faced by students. Give your opinion regarding the importance and the effect of each element. 5 means the element is influential. 
Linguistic/ semantic elements of the wordproblem

2. The existence of irrelevant information

3. Relevance of the context of the problem to the student's life

4. Student's familiarity with the setting/ environment of the problem

5. Vocabulary used in the word-problem

6. Structure of the word-problem (direct/passive voice)

7. The existence of lengthy sentences

8. Others: please state here

- The following are some elements related to individual students that have been reported in the literature as factors behind the difficulties faced by students. Give your rating of the importance and the effect of each element. 5 means the element is influential.
1. Disposition toward mathematics

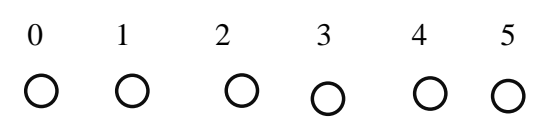

2. Patience and persistence to solve the problem

3. Self-confidence

4. Ability to find key word

5. Ability to find hidden information

6. Ability to break the problem into informative parts.

7. Student's English language proficiency

8. Students do not read the problem carefully

9. Others: please state here 


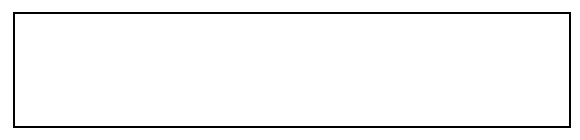

- The following are some factors that might affect a teacher's decision to apply wordproblems. Give your opinion regarding the importance of each factor. 5 means the factor is influential.

1. Number of students in the class

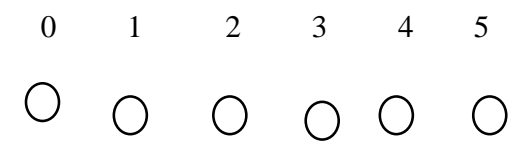

2. Allocated time of the lecture (morning/ afternoon)

3. Duration of the class

4. Appropriateness of the class setting

5. The academic level of students

6. Others: Please state here

\section{APPENDIX II}

\section{SEMI-STRUCTURED INTERVIEW PROTOCOL}

1- In your opinion, why students find word-problems challenging?

2- What are students' limitations that might affect their ability to work on word-problems?

3- What kinds of mathematical skills students need to be able to solve word-problems?

4- In your opinion, what is the most challenging part/phase of solving a word-problem?

5- Some might argue that if a student is studying mathematics in a language different than his/her mother tongue then they will face difficulties with word problems. Give us your feedback on this issue.

6- Let's talk about your pedagogical style, what kind of strategies you use to reinforce student's ability to solve word-problems?

7- Have you taught in the school level? If yes, do you find this issue different for high/middle school students?

8- Have you taught outside KSA? If yes, can you compare your experience with solving word problems in KSA and outside KSA. 